\title{
Toward a simulation of minibuses in South Africa
}

\author{
Andreas Neumann \\ Daniel Röder \\ Johan W. Joubert \\ Technische Universität Berlin ${ }^{a}$ Senozon Deutschland GmbH University of Pretoria
}

\begin{abstract}
After private cars, minibus taxis are the most common transport mode in South Africa. Especially for low-income citizens living in townships, minibus services are often the only possibility for mobility. Despite the great importance of the mode, there is very little knowledge of routes, fares, and the number of minibuses. Hence, it is difficult to simulate and to understand the influence of this mode on other modes and on transport planning in general. This article presents the development of the first "close-to-reality" minibus supply model based on demand and street network only. The approach adopts the survival-of-the-fittest principle, using a coevolutionary algorithm that is integrated into a microscopic multi-agent simulation framework. The successful application of the approach to a large-scale, real-world scenario in the Nelson Mandela Bay Area Municipality in South Africa shows that it is able to identify the main minibus corridors as well as to find robust service coverage in lower-demand areas. The resulting minibus supply model can then be used for planning purposes (e.g., to investigate aspects of strategic, operational, or regulatory changes).
\end{abstract}

\section{Introduction}

The term paratransit has two meanings when referring to transport. The first describes a kind of transport specially fitted to the needs of elderly or physically handicapped people. This paper, however, deals with the second meaning: a spatio-temporally flexible mode of transport ranging from taxis up to bus lines (Roos and Alschuler 1975). This means that either the routes (space) or schedule (time), or both, are flexible. Formal transit would typically have fixed routes with fixed schedules. On the other end of the spectrum, dial-a-ride services are flexible in both their routes and schedules. In most cases, this is a user-demand-oriented mode of transport mainly used in cities of the developing world. Although paratransit shares some underlying principles with demand-responsive transit (DRT) systems, it can be distinguished by the way organization takes place. DRT systems heavily rely on a supervising level (controller), such as an agency or government authority, that allocates vehicles to individual trips or collective rides (e.g., ENEA 2004; Jokinen et al. 2011).

Many paratransit services are complementary to scheduled public transport. According to Cervero (2000), in most instances, paratransit services compete with rather than complement formal bus and rail services. This happens because paratransit usually emerges and evolves when little or no formal transit is provided by the relevant government.

In South Africa, as in many developing countries, paratransit takes the form of mini- and midibuses that are privately owned and provide communal transit services along semi-structured routes. Commuters often hail the vehicles along the route, as there is seldom a formal stop infrastructure. The services also do not follow fixed schedules. Such a service-minibuses with fixed routes but without fixed schedules-is often called a jitney service. This paper will use the term "minibus service" and refer to the operator as a "minibus operator" with the understanding that the jitney/minibus service is one of many possible paratransit services.

\footnotetext{
${ }^{a}$ neumann@vsp.tu-berlin.de Copyright 2015 Andreas Neumann, Daniel Röder, and Johan W. Joubert.

doi: http://dx.doi.org/10.5198/jtlu.2015.390

ISSN: 1938-7849| Licensed under the Creative Commons Attribution - NonCommercial License 3.0.
} 
The lack of routes and schedules often creates a semi-chaotic perception of the mode, resulting in the mode being viewed as a necessary nuisance that should be formalized. Especially in developing countries, paratransit remains largely unregulated, which often results in factions within the industry fighting, literally, over lucrative routes.

In the absence of documented routes and schedules, it becomes very difficult-if not impossibleto include such a mode into planning models. Instead, it is dealt with qualitatively in an after-the-fact manner. When transport planners are faced with developing decision-support tools, they often omit paratransit since it makes up a very small percentage of service to the total road user population. In a country like South Africa, however, paratransit is the dominant mode of transport when all trip purposes are considered. Omitting it from formal planning would render the planning model inadequate for comprehensive and inclusive decision making. The main challenge so far has been the inability of equilibrium assignment models and activity-based planning tools to cater to the dynamics of paratransit.

To our knowledge, this paper presents the first (behaviorally rich) implementation of the dynamic and evolutionary nature of jitney-like paratransit services in a formal, large-scale transport model. To achieve this, we extend and build on an existing large-scale agent-based transport model in South Africa. More specifically, we employ the autonomous nature of agents to emulate the unique decisionmaking process inherent in the minibus industry, and we introduce new agents to represent different stakeholders. Transport planners can then consider conducting policy analyses using the model.

The structure of this paper is as follows: the next section will give a short introduction to the jitney-like minibus system. Although the case of South Africa will be used, it is extendible to many developing countries. Section 3 describes the proposed minibus taxi model and how it is incorporated into the agent-based setting. The model is then applied to a large-scale scenario covering the Nelson Mandela Bay Municipality (NMBM) in Section 4. The paper concludes with an analysis of the scenario's outcomes as well as an outlook for the model and possible further improvements.

\section{The minibus industry in South Africa}

For a more international discussion on paratransit in various developing countries, the interested reader is referred to Cervero and Golub (2007). In this section, we provide a more focused discussion on the South African case. The literature on the minibus mode is quite sparse and includes early works by McCaul (1990), Pirie (1992), Khosa (1994), and Dugard (1996, 2001).

One of the legacies of the former apartheid regime in South Africa still evident today is the location of informal and semi-formal townships on the periphery of cities and towns. These settlements are mainly occupied by low-income citizens who generally cannot afford private vehicles for their daily commute.

In the absence of reliable formal transit provided by the apartheid government, the minibus industry emerged as the dominant mode of transit serving these poor, mainly black, peripheral townships. Over the past three decades, it has evolved to account for three-quarters of all transit trips. An entire minibus industry emerged over time and forms part of what is colloquially known in South Africa as the second economy: a cash-based, unsubsidized industry that is fairly self-regulatory with very little documentation of fare structures, routes, or schedules-the last not really existing at all.

For a detailed background on the rise of the minibus taxi industry, as it is known in South Africa, the reader is referred to Joubert (2013). Suffice to say that a loophole in legislation allowed a driver to transport up to eight passengers, for gain, before being classified as a bus, which is subject to much more stringent regulations and operating licenses.

When considering only work-related trips, the minibus is only rivaled by the private car. Considering all trip purposes, however, it even exceeds the private car. Unfortunately, the mode is in direct 
competition with formal (subsidized) transit provided by the government. There are two types of competition. First, there is the head-to-head competition with conventional public transport buses along popular routes, effectively duplicating the routes. Minibuses arrive at the stop just before the conventional bus, taking away passengers by offering a faster, albeit more expensive, trip. Second, there is the complementing type of competition. This happens if headways on the fixed-schedule bus are too long and the minibus fills in the gap, shortening the effective waiting time. For a more comprehensive description of the characteristics and underlying principles of paratransit systems, and minibuses in particular, the reader is referred to Neumann and Nagel (2012) and Neumann (2014).

\subsection{The industry structure}

Although it was common during the 1980s for drivers to own their vehicles, most minibus drivers nowadays are employed by vehicle owners. Owners have organized themselves into associations that, in turn, nominate representatives to provincial and national councils. The South African National Taxi Council (SANTACO) has long been the single body with which the government interacts. But, as argued by Woolf and Joubert (2013), the minibus industry remains a heterogeneous grassroots operation and not a homogeneous body, as it is often perceived. The industry's amorphous structure has contributed to the popular public view that paratransit, in its current form, should be formalized. To this extent, Schalekamp and Behrens (2010) and Venter (2011) review the unsuccessful attempts by government to get a tighter handle on the industry.

For the purpose of this paper, it is necessary to elaborate more on some of the key stakeholders in the industry and the context within which they operate daily.

\subsubsection{Associations}

Taxi associations form when taxi owners serving a similar geographic region organize themselves to protect their routes. Route operating permits are issued by provincial governments, and the permits only vaguely describe the routes based on their origin and destination ranks (terminal). It is up to the associations to negotiate their respective "turfs" amongst themselves. Conflicts often arise between associations when there is an overlap between the agreed operating areas. This typically happens when the ranks of local and long-distance services coincide and when independent drivers provide services without association approval. The latter are referred to as pirate taxis and remain a headache for the minibus industry.

\subsubsection{Owners}

Minibus owners, knowing their operating routes, can roughly calculate an expected daily income, referred to as the check-in amount. It then becomes the goal of the minibus driver to earn the check-in amount for the owner. Any additional income is kept by the driver.

If owners manage their businesses well, they may be capable and choose to extend their business by acquiring more vehicles. First, an owner must acquire a valid operating license from an association. Finding a driver for the vehicle is not often a problem in a country with unemployment in excess of 25 percent. Irregularities in the issuing of operating licenses mean that even in the presence of a moratorium, new licenses are still being issued. As a result, there is usually an oversupply of minibuses in the system, and if the business of an owner is not successful, the owner may eventually pull out and sell one or more of his or her vehicles. 


\subsubsection{Drivers}

Minibus drivers earn income from fixed, route-based fares. That is, the fare for a route is set based on origin and destination and does not depend on where along the route the passenger boards or alights. About 60 percent of all boarding and alighting happens along the route. From the fares earned, drivers must pay for all fuel expenses and fines (a regular occurrence), while owners are responsible for vehicle maintenance. Once the morning peak demand is over, the majority of drivers will cease service since it incurs costs without yielding certain income. Drivers will typically park near the busy areas and resume their services as demand increases again in the afternoon.

\subsubsection{Fares}

Fares are usually calculated to cover the expenses of a complete journey. That is, regardless of whether a passenger enters a vehicle close to the origin or to the destination, the fare is always the same. An example for that is given by the Transport Plan of Johannesburg (City of Johannesburg 2004, p.99). However, the fares mentioned in the Transport Plan differ seriously from the authors' personal experience. For example, in August 2013, a short trip to Pretoria Central of $4 \mathrm{~km}$ may cost ZAR 14 (South African Rand, equivalent to approximately EUR 1.06 in August 2013), even though commuters starting their journey at the origin, Mamelodi, which is $25 \mathrm{~km}$ away, also pay ZAR 14. Fares calculated on the basis of the numbers derived from the Transport Plan amount to ZAR 2.6 for the short trip and ZAR 4.0 for the longer trip.

\subsubsection{Commuters}

Since routes are not documented, commuters require tacit knowledge of how to navigate the network. This knowledge is acquired through word of mouth. In the absence of formal stops, and because the minibuses are not signed in terms of their routes, commuters use an intricate and location-dependent set of hand signals to hail a taxi (Woolf and Joubert 2013). The hand sign indicates to an approaching taxi what the commuter's desired destination is. If that destination coincides with the driver's destination and agreed route, the driver will stop for the commuter to board, capacity permitting.

\section{Model description}

The model used in this paper enhances the multi-agent simulation MATSim (2013) with a more sophisticated version of the minibus model used in Neumann and Nagel (2012, 2013). In contrast to demand-responsive transport systems (DRT) — which tend to find a system optimum because the services are cooperating and thus solve one system-wide instance (Cortés 2003; Fernandez et al. 2008; Pagés et al. 2006) —each operator in this model evolves according to its own optimization procedure. This is related to co-evolution and evolutionary game theory (e.g., Arthur 1994; Drossel 2001; Hofbauer and Sigmund 1998; Palmer et al. 1994). Synthetic minibus operators increase or decrease their service frequencies by adding or removing vehicles, depending on each individual line's fitness. When no vehicle is left for a line, the line dies out. In this paper, the focus lies on the enhancements of the minibus network and only a brief overview of the software tool MATSim is given. For an in-depth description of MATSim, the reader is referred to Balmer et al. (2005) or Raney and Nagel (2006). The public transport capabilities of MATSim are described in general in Rieser and Nagel (2009) and Rieser (2010) and with a focus on its application in Neumann and Nagel (2010). 


\subsection{A brief overview of MATSim}

In MATSim, travelers of the real system are represented by individual agents. Agents typically have several plans that encode, among other things, their desired activities during a typical day as well as the transportation mode per leg. Currently, the private car and public transport are physically simulated. Other modes are not physically simulated - the travel time of one leg depends on the beeline distance and an average speed only. At each time of the simulation, only one plan is selected for execution. Agents can react to their synthetic reality (traffic flow simulation) through an iterative loop that has three steps:

- Traffic flow simulation: All selected plans are simultaneously executed in the simulation of the physical system.

- Scoring: All executed plans are scored by a utility function.

- Learning: Some agents are allowed to modify their plans with different degrees of freedom. In terms of this work, only the search for new routes is allowed.

The physical simulation of MATSim executes the selected plan of each agent. Since all agents compete for the same limited resources, the execution may deviate from the intended plan. For example, an agent can be stuck in a traffic jam, causing that agent to arrive late at his or her next activity. In the case of public transport, drivers also try to follow their plan and compete with other agents/drivers on the road network. Agents desiring to use public transport as passengers will have to wait for the next departure if the vehicle passing by has no capacity left. If there are no further departures, the agent is also stuck.

All executed plans are evaluated by a utility function, which in this paper encodes the perception of travel time for the following modes: car, train, bus, minibus, and walking. For minibus users, the utility function also accounts for waiting, access, egress times, and line switching.

\subsection{The minibus model}

The minibus service is implemented as a part of the public transport system of MATSim (Rieser 2010). Thus, minibuses face the same restrictions derived from the network (i.e., allowed speed and capacity constraints) as other public transport vehicles and private cars. Minibuses are delayed by a) other vehicles regardless of type and $b$ ) boarding and alighting passengers. Due to a lack of data on origins, destinations, and the actual routes served, an evolutionary algorithm is used to create the minibus network from scratch.

At the beginning, each minibus operator starts with one line that serves one circular route/minibus service. The route is determined by two randomly selected stops and the fastest path connecting both stops in an empty network. The random draw for a stop is weighted by the number of activities in its proximity in order to attract newly found operators to those more promising areas. Initially, this first route is operated from a randomly selected start time to a randomly selected end time. The initial number of minibus operators and the number of minibuses per operator (serving the first route) can be configured.

In spite of reality, minibuses are assumed to run without breaks during their time of operation and will depart immediately after arriving at their terminus. Minibuses are allowed to pick up passengers at every intersection. Minibuses can overtake each other and other public transport vehicles at stops. A fully loaded minibus will not try to pick up additional passengers and instead will proceed as quickly as possible to the next stop, determined by a passenger's desire to alight. A minibus with empty seats will ask the waiting agents at each stop on its route if they want to enter. The questioned agent will enter the vehicle when: 
- The vehicle is running on the correct mode (i.e., an agent planning to board a bus will not enter a minibus).

- The vehicle is serving the desired destination/stop.

- The offered travel time is less or equal to the agent's planned travel time.

Thus, agents are not forced to use planned routes but are forced to use routes heading toward their destination within an acceptable time.

Due to the evolution of the minibus system, an adjustable set of agents will be allowed to find new routes after each iteration. For that, an independent instance of MATSim's current public transit passenger router (Rieser 2010; Rieser and Nagel 2009) is used. In contrast to Neumann and Nagel (2012, 2013), agents are only allowed to search for a new route within the mode included in their initial plan. The router searches the time-minimizing connection for each agent using the corresponding mode with respect to access and egress walks, waiting time, and transfers. The fare calculation is omitted in route searching due to a lack of detailed information on fares, especially for minibuses.

In spite of the different behavior of minibuses compared to formal public transport, route planning by the passenger is similar to a schedule-based transit assignment. Agents include minibus trips in their plan assuming that there will be a certain minibus at a certain stop at a certain time. Especially because minibuses ignore the timing of their schedule and tend to drive as fast as possible, a minibus may be running far off its schedule. However, for South African minibus services running at high frequencies, this is not a serious issue since the agent will just take the first approaching minibus heading to the desired destination.

\subsection{Scoring of the minibus operators}

The operator scoring in this paper is the same as used in Neumann and Nagel (2013). At the end of each day (iteration), the operator calculates the revenue generated by each of his or her routes and the expenses related to these routes. Revenue is generated by collecting fares. The fare system allows for lump sums, distance-based fares, and combinations of both. Expenses consist of fixed costs and distance-based costs. Fixed costs cover expenses related to the vehicle (e.g., official operating license and driver). Distance-based costs (e.g., fuel) are summed up for each kilometer traveled by the operator's vehicles.

The total score of one operator/line can be seen as the operator's (net) cash flow. Profitable operators end up with a positive cash flow, and non-profitable lines end up with a negative cash flow. At the end of the iteration, the cash flow is added to the budget of the operator.

\subsection{Optimization process}

Since a minibus line is operated by one operator, each operator tries to improve his or her own line. There is no explicit coordination or cooperation between the operators, except for the fact that an agent using minibus can transfer to a different minibus line or route. Different operators together can thus form a hub if this emerges from the optimization process, but otherwise they are engaged in competition (e.g., Axelrod 1984). If two different operators ply a similar route, the operator providing a slightly better route (e.g., without additional transfers in between) can oust the other one from the market.

Minibus operators optimize their services in parallel with the agents' adaptation process. In every iteration, the following happens:

1. The operators modify their routes as described below and publish their (pseudo-)schedule valid for the current iteration. 
2. A randomly selected set of agents obtains a new route based on that (pseudo-)schedule, computed as described earlier. The other agents remain on their existing route for this iteration.

3. The traffic flow simulation is run with those minibus operators and passenger agents.

That is, for the present paper, the passengers do not optimize beyond what is described in item 2. This means that they do not in fact react to the actual schedule or to congestion, including denied boarding. Opening more degrees of freedom for the agents' replanning is problematic as a) the available data (e.g., fares) is rather sparse, b) the interaction between operator and traveler becomes more complex and unpredictable, and c) the decision regarding which mode is used is based on intrinsic motivation that has not yet been parameterized.

At the beginning of each optimization step, operators balance their budget to zero by selling or buying minibuses. An operator not able to balance its budget is shut down and another one is initialized. Since the budget depends on the profit of a line, the number of minibuses owned by an operator should fit the market restrictions (i.e., the cash flow is balanced and the number of minibuses becomes stable).

If the operator is not shut down, he or she can further try to optimize his or her current line. The operator chooses one of his or her existing routes by using a random draw based on the number of minibuses serving that route, copies the drawn route, and alters one of the following route attributes:

- The time of operation. An operator can increase the time of operation by changing the time of the first or the last planned departure. Alternatively, an operator can decrease the time of operation by analyzing the demand of the last iteration. The start time is then set to the time of the first passenger boarding one of his or her minibuses, and the end time is set to last passenger alighting. This can compensate for slack periods, minimizing the expenses of empty minibuses circulating.

- The actual stops served by the route. An operator can decide to serve an additional stop. This additional stop will be drawn from a set of unserved stops within a specified distance/area around the existing route. Now the chosen stop can be added before the first stop or injected in between the two nearest existing stops of the route. Similar to the reduction of the time of operation, the operator can analyze the demand flow of the stops served. Stops not generating enough revenue to cover their related costs can then be discarded. The remaining stops form the new route.

Since operators assess their routes by calculating individual scores for each route, they know which routes add most to the success of the line and which ones burn money. At the end of the optimization, operators can shift minibuses from less profitable routes to more profitable ones. If a route's last minibus shifts away, it will immediately cease operation and is removed. More minibuses serving a route directly translate into a higher frequency of that route. Eventually, each route of an operator has a similar score per minibus. Thus, the number of minibuses on a route represents the importance of that route to the operator and thus gives a good estimate for deciding which routes to improve further.

\section{Scenario}

The synthetic population used in the simulation of the NMBM is generated using two steps. Firstly, we use iterative proportional fitting (IPF), similar to the implementation by Müller and Axhausen (2011). Using the Census 2001 population as source data, the synthetic population is fitted on both the household and individual level. 
Table 1: Characteristics of the generated population and the used sample.

\begin{tabular}{rrr} 
& Complete population & $1 \%$ Sample \\
\hline Persons [\#] & $1,164,150$ & 11,498 \\
Share of females [\%] & 52.5 & 52.2 \\
Share of males [\%] & 47.5 & 47.8 \\
Average age [years] & 27.7 & 27.1 \\
Car trips [\#] & 356,208 & 3574 \\
Minibus trips [\#] & 920,722 & 9261
\end{tabular}

Secondly, travel demand for the population is based on a 2004 travel survey that includes a 24hour trip diary, covering approximately 1 percent of the population. For each household surveyed, a questionnaire was completed that covered general household information such as number of persons in the household, employment status of the household members, number of cars available, and overall household income. For each member of the household, individual travel information was gathered, among which was a detailed description of the individual's activity chain for the day. Each activity was described using a predetermined activity type, start and end time, and the mode connecting the different activities in the chain.

Every individual in the synthetic population is assigned an activity chain sampled from the survey chains. The sampling ensures that the chain is from an observed individual with similar characteristics in terms of employment status, household size, age, and household income. From all households a 1 percent sample is randomly drawn whose members form the synthetic population for this scenario. The complete synthetic population and the 1 percent sample are characterized in Table 1.

Figure 1 shows the distribution of activities within the NMBM and the high spatial diversification of home locations (Figure 1a) and working places (Figure 1b). The home locations are mainly in Kwazakele, Zwide, New Brighton, Gelvandale, Westend, and Kwanobuhle. The working places are mainly located in the city center between N2, Cape Road, and Settlers highway and in "Alexander Park Industrial" in Uitenhage (north) where the Volkswagen South Africa assembly plant, among other large production facilities, is located.

The network is generated from OpenStreetMap (2012) data and contains 39,507 links.

The model is initialized with 35 minibus operators, each with an initial fleet size of 21 minibuses. Since the underlying traffic flow simulation simulates only whole passengers, the typical South African minibus capacity of 16 passengers cannot be reduced to 1 percent (i.e., 0.16 passengers). Instead, the capacity is set to 2 passengers per minibus. While this exceeds the actual capacity of a " 1 percent minibus," it allows us to simulate loads different from "completely empty" and "no capacity left." Operators are allowed to buy new minibuses for a price of 1000 monetary units. They can sell minibuses for 250 monetary units each. Operating a minibus costs the operator 10 monetary units per day/iteration and 0.25 per $\mathrm{km}$. Operators earn 3 monetary units per passenger entering a minibus, irrespective of the distance the passenger travels. Note, as shown in section 2 , fares are fairly unknown. Thus, the fare structure for the experiments was chosen to deliver reliable results in terms of passenger flows and route structure. Newly found operators have four iterations to break even. Operators are allowed to create new routes until the end of iteration 150. Buying and selling vehicles is allowed until the simulation ends. Minibus stops are located on all links with a speed limit of $80 \mathrm{~km} / \mathrm{h}$ or below.

In contrast to other applications of the minibus model (e.g., Neumann 2014; Neumann and Nagel 2013), we omit the physical simulation of formal public transport due to a lack of consistent data. Thus, only cars and minibuses are physically simulated. 


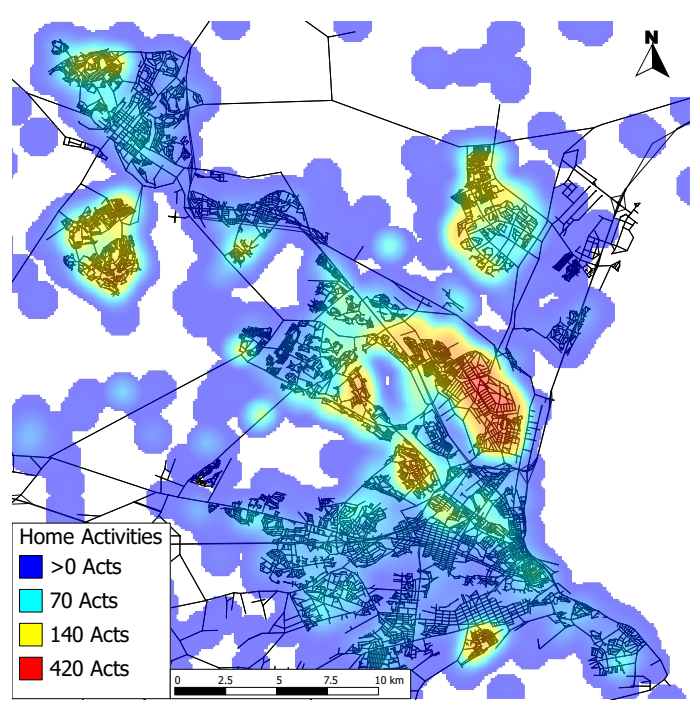

(a) Distribution of home activities

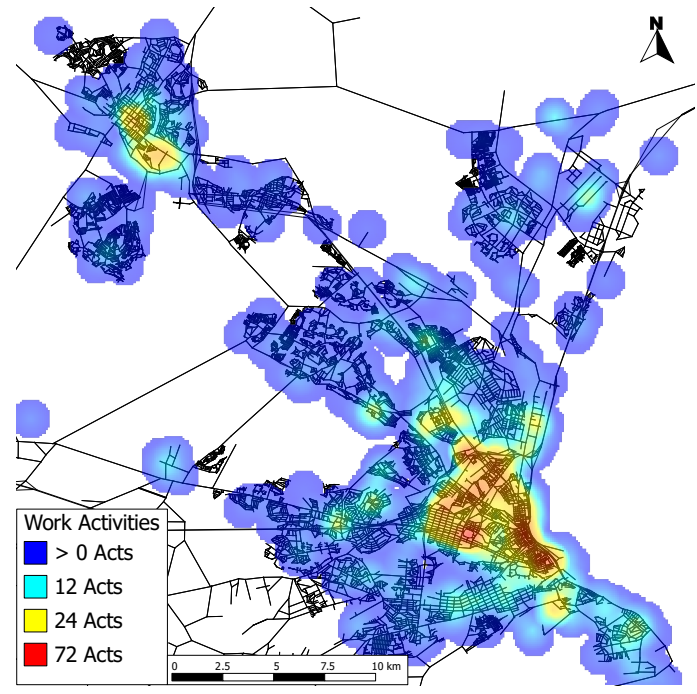

(b) Distribution of working activities

Figure 1: Heat maps showing the distribution of home and work activities.

Passenger agents have three plans in their choice set. In each iteration up to the end of iteration 200 , a randomly chosen set of 40 percent of the agents is allowed to search for an alternative route within their transport mode. Agents will then stick to that route for the following iterations until they are selected for rerouting again. Based on the authors' experience, transferring to a different minibus results in an additional waiting time because the next vehicle will only depart when it is fully loaded. Since vehicles in the model depart immediately, the delay is simulated within the router by setting the penalty for a line switch to an additional 30 minutes of travel time.

We test for convergence of the system by the same standards as in Neumann and Nagel (2012, 2013) - i.e., we analyze the number of trips performed, the number of minibuses put into service, and the average score of the population.

\section{Results}

In the beginning of the simulation, there is only a randomly drawn minibus supply. As expected, this supply is neither able to serve the existing demand nor does it fit the real system (as far as information is available). Thus, a large number of agents will be late or even never reach their destination. One might notice two effects for the agents' departures (Figure 2a) and arrivals (Figure 2b). Firstly, both numbers increase over the iterations with a declining effect in higher iterations, especially iteration 150 onwards. This results from the fact that the evolution of the minibus system is reduced to the capability of buying and selling vehicles.

Secondly, the histogram shifts from the right to the left toward earlier departures and arrivals. This effect might be much more clearly noticed viewing Figure $2 c$, which shows the agents en route. In the very first iteration, approximately 830 agents do not reach their planned destination. This results from spillover effects induced by all agents choosing the best option from the schedule. In iteration 150, the number of agents stuck decreases to approximately 350 due to operators adapting to the supply by putting more minibuses into service and thus increasing the overall system's capacity. Due to the highly competitive minibus market with operators becoming bankrupt and the foundation of new operators, an agent's current plan might not reflect the latest changes in the minibus network and thus be invalid. 
At the final iteration (300), exactly 191 agents are left who never reach their destination. The majority, 169 agents, try to use a minibus route that does not exist anymore- the route has been dropped by the operator. The remaining agents miss the last minibus' departure (13) or cannot be served because all minibuses passing by are fully occupied (9). As noted earlier, within the last 100 iterations, operators can only change the capacity of their routes by reallocating vehicles or by dropping routes entirely. A route is dropped when the operator regards it as unprofitable (e.g., due to insufficient demand). If an agent's choice set contains only dropped routes, that agent will inevitably be stuck. That is, the minibus model cannot guarantee operators serving all of the population. Instead, operators serve as much of the population as they can afford.

\subsection{Minibus service coverage}

As illustrated in Figure 3, the majority of the urban area has access to minibus services within a walking distance of less than $500 \mathrm{~m}$. Passengers traveling to or from areas with a low density of activities need to accept longer walking distances to and from the minibus system (compare Figure 1). Note that for South African cities, access trips of up to $2.5 \mathrm{~km}$ are considered normal. For example, figures from the Department of Transport (DoT 2005, p. 38) state that about 74 percent of households have access to minibus/taxi services within 15 minutes of walking and an additional 13 percent of households can access a minibus/taxi service within 15 to 30 minutes.

To test for the sensitivity of the model, two additional runs of the same scenario with the same configuration but a different random seed are conducted. Their resulting catchment areas (smaller figures shown in Figure 3) show a similar level of accessibility with only minor differences in lowdemand areas. High-density remote areas like the Walmer-Township or Khaya Mnandi are covered in all three scenarios with walking distances of less than $500 \mathrm{~m}$.

The analysis of access walks from the home location to the minibus system shows that more than 85 percent of minibus users walk less than $1000 \mathrm{~m}$ to access the system (see Figure 4). This roughly matches the sparse data available from the South African National Household Travel Survey (DoT 2005) for a "typical metropolitan area" in South Africa. The travel time data included in the survey is transformed in distance classes assuming an average walking speed of $4 \mathrm{~km} / \mathrm{h}$. The authors are not aware of any data sets describing the area of the NMBM in particular. However, the simulation results fit the data available for a distance of $2000 \mathrm{~m}$ and only marginally overestimate the accessibility for a distance of $1000 \mathrm{~m}$. Overall, the deviations are regarded as negligible.

\subsection{Minibus corridors and passenger volumes}

The final minibus network consists of 87 routes operated by 25 individual operators. Operators can be classified according to the type of service they provide. The classification shown in Figure 5a distinguishes between local operators accessing neighborhoods through a dense (ramified) network, feeder lines providing service from nearby townships to the city's center, and long-distance services connecting the northwestern part of the research area (Kwa Nobuhle, Uitenhage) to the city. Since the routes of all operators can overlap each other, the sum of all routes forms the minibus network. Especially from the point of view of the simulated passenger, it does not matter which type of service a minibus belongs to as long as it has capacity left and serves the destination the passenger asks for. Thus, the minibuses and routes of different operators form a combined minibus service. In consequence, trunk roads with multiple routes running at a high frequency provide higher-quality service due to more frequent service and a higher probability that the passenger will be picked up. Figure $5 \mathrm{~b}$ illustrates the number of passengers served per road segment regardless of the actual operator used, with Uitenhage/Commercial Road having the highest demand of more than 50,000 trips per day when scaled up 


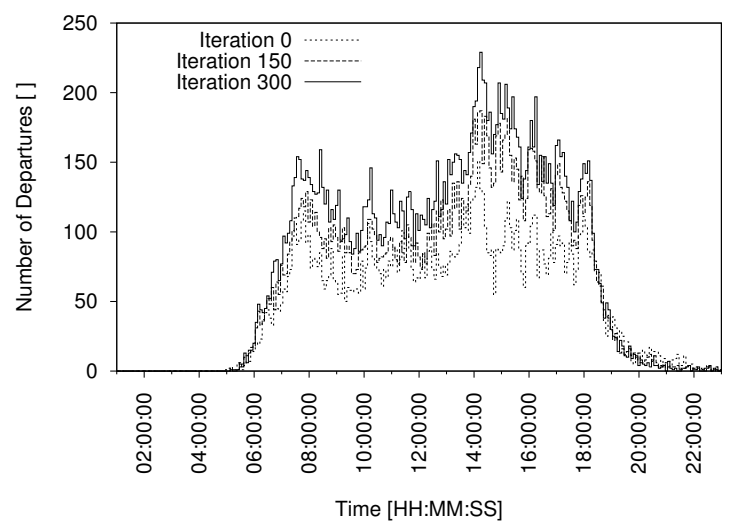

(a) Number of departures of agents

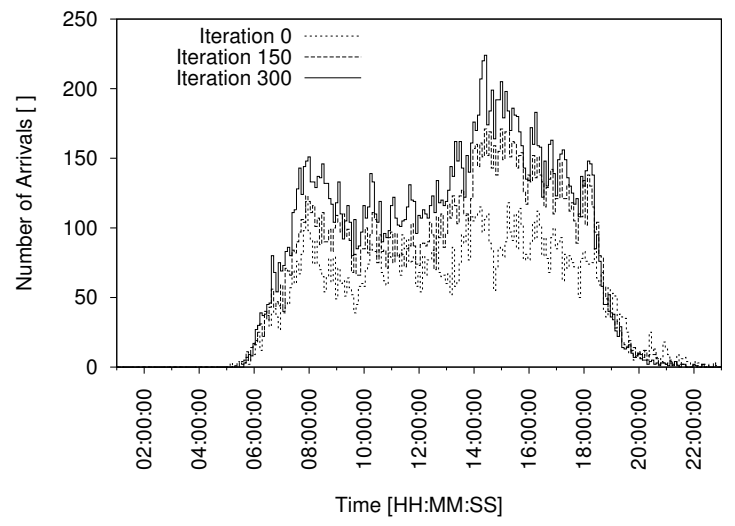

(b) Number of arrivals of agents

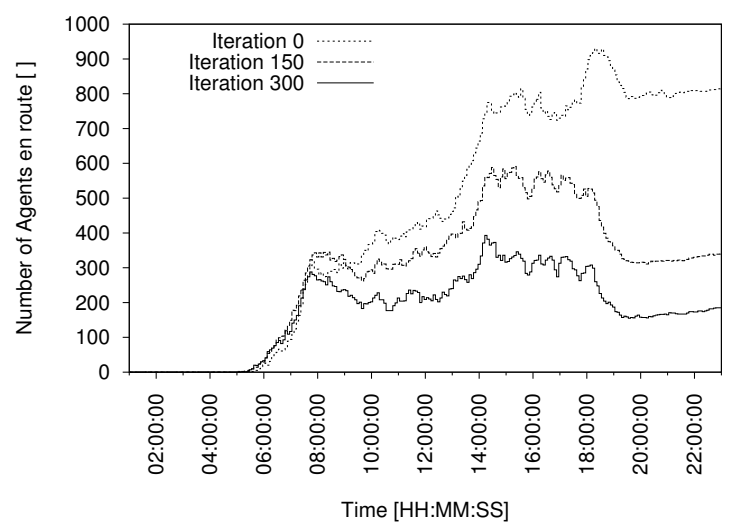

(c) Number of agents en route

Figure 2: Leg histogram evolution of the taxi system. 

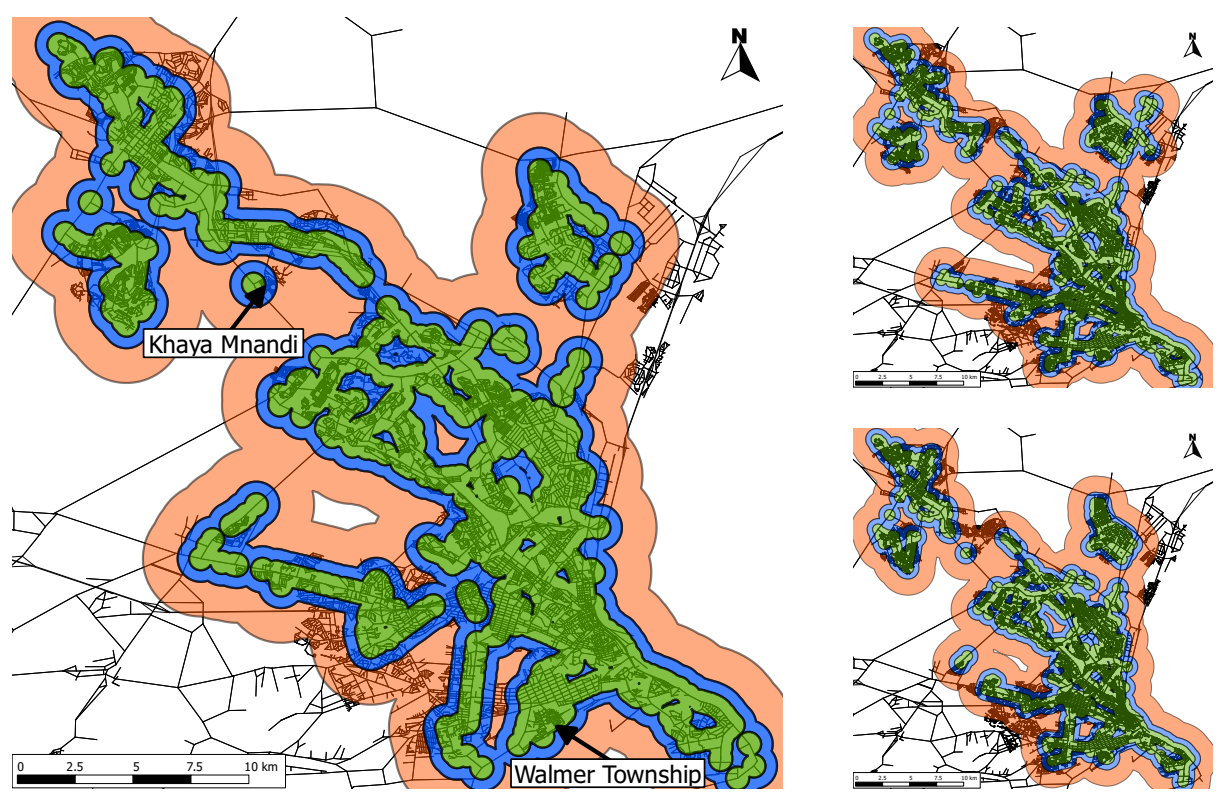

Figure 3: The minibus services' catchment area of less than $500 \mathrm{~m}$ (green), $1000 \mathrm{~m}$ (blue) and $2500 \mathrm{~m}$ (orange). The smaller figures show results from sensitivity runs.

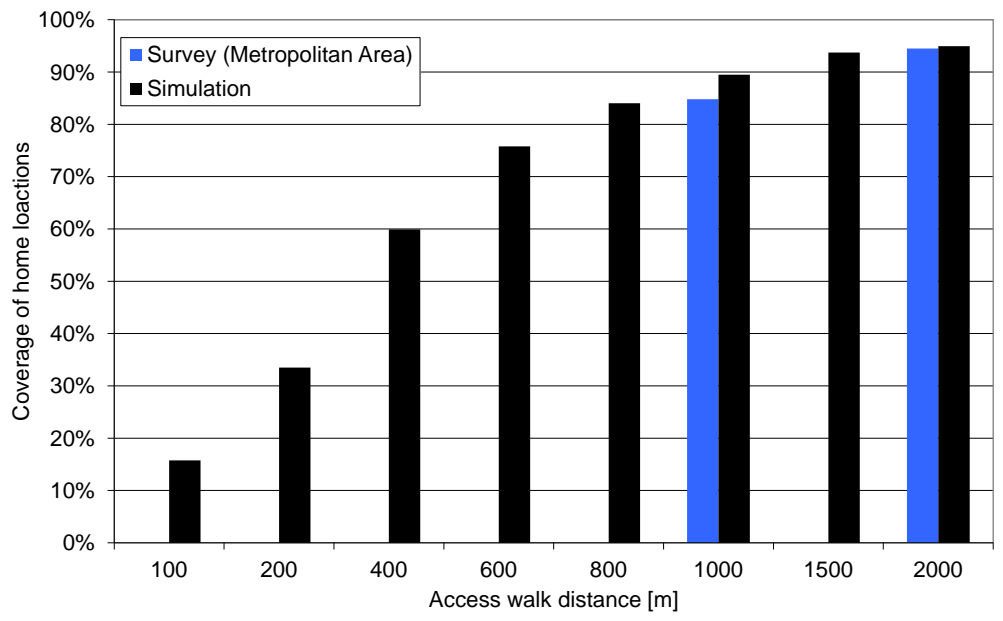

Figure 4: Minibus service access walk distance distribution from home locations.

to 100 percent. Overall, the average waiting time for boarding a minibus is 4 minutes 20 seconds in the model, which matches the 5 minutes estimated by local expert knowledge.

For validation purposes, count data of minibus passengers are derived from the Transport Plan (SSI Engineers and Environmental Consultants 2011). As Figure 6 shows, the figures of the simulation compared to the provided count data reveals a large discrepancy in numbers. However, the count data stated in the Transport Plan derive directly from a simulation with no further explained input data. Since the demand of our scenario originates from a comprehensive household survey, the number of trips and locations stated in the survey and thus in our model are considered more reliable than the figures stated in the Transport Plan.

One might notice that the "Cape Rd" count station shows a simulated value of zero, whereas the Transport Plan states an albeit low value. Sensitivity tests show that the road segment of that count 


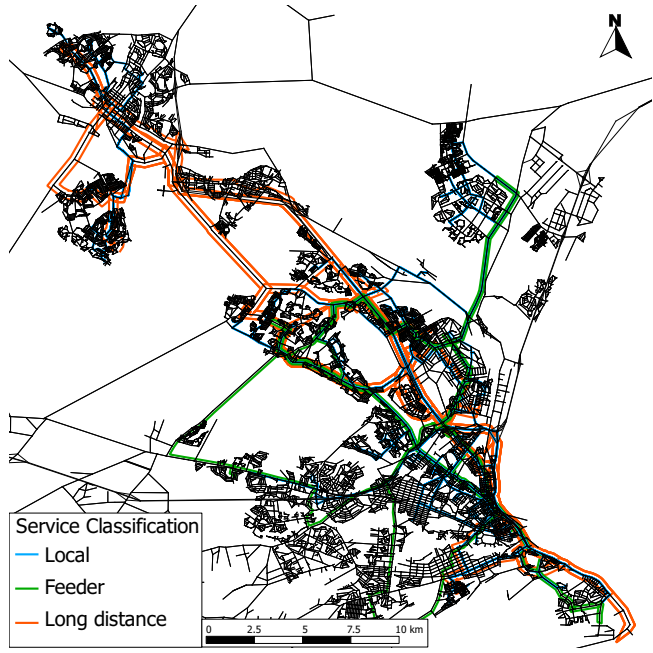

(a) Lines operated

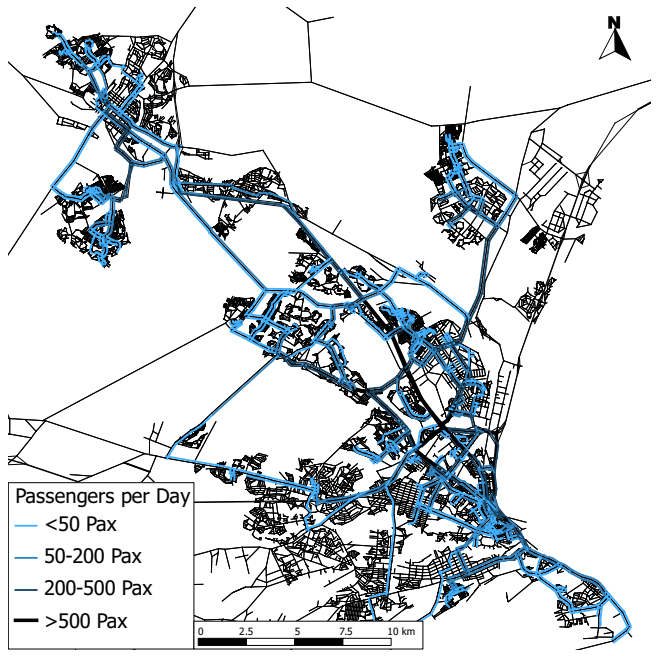

(b) Passenger volumes

Figure 5: The final minibus system.

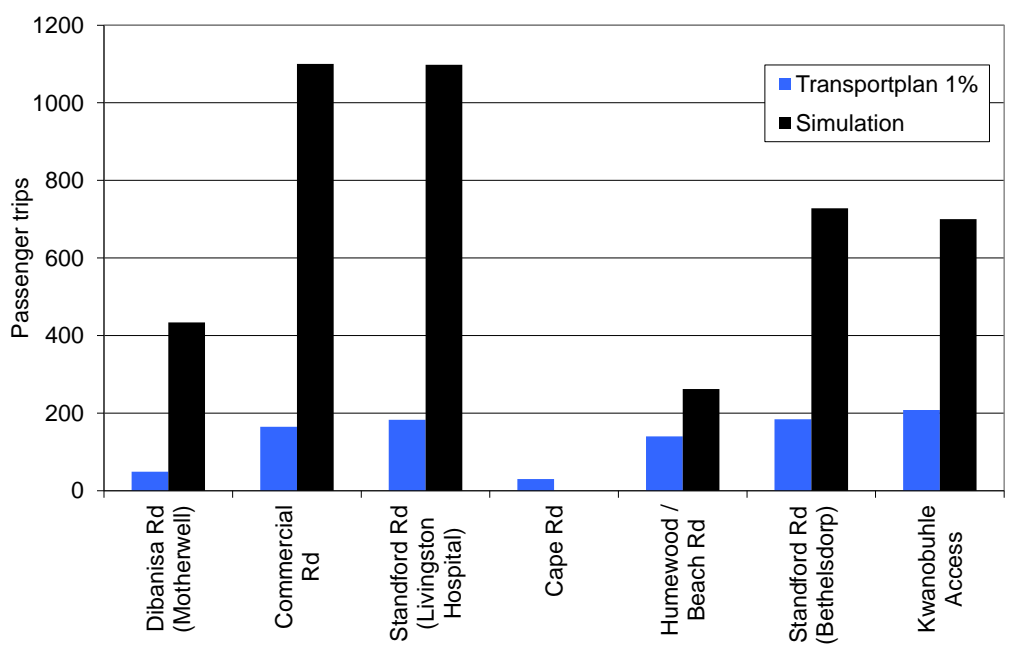

Figure 6: Comparison of minibus passenger count data.

station is served only occasionally. This is mainly for two reasons. Firstly, the surroundings of the count station feature only a few activities, making it not very attractive for operators to serve this area directly. Secondly and more important, due to the count station's location at the edge of the minibus' service area the probability of a minibus route passing by is very low. Furthermore, the dense network in this area provides plenty of opportunities to circumvent the count station itself by making smaller detours.

\subsection{Boarding and alighting of passengers}

As illustrated in the leg histograms shown in Figure 2, the population using the minibus system features two demand peaks, one in the morning from 0700 to 0800 and one in the afternoon between 1400 and 1500. In addition, the activities of the most common home-work trips of minibus users form spatially 


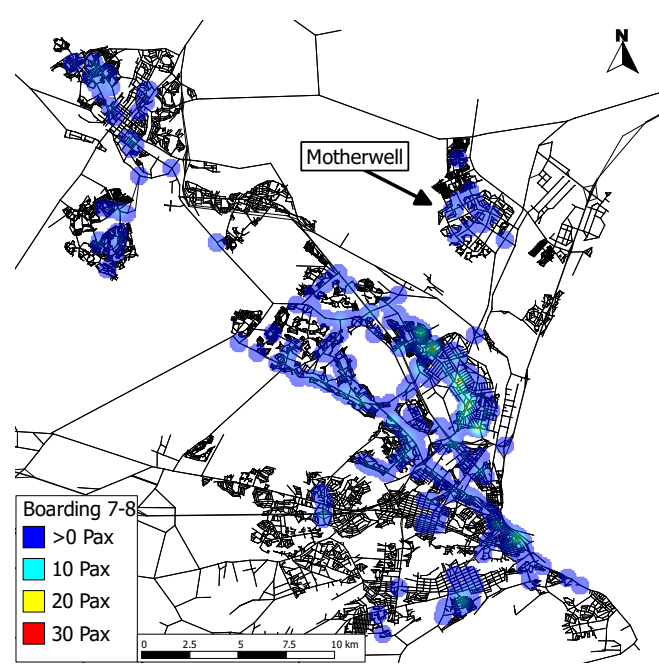

(a) Minibus boardings between 7 and 8

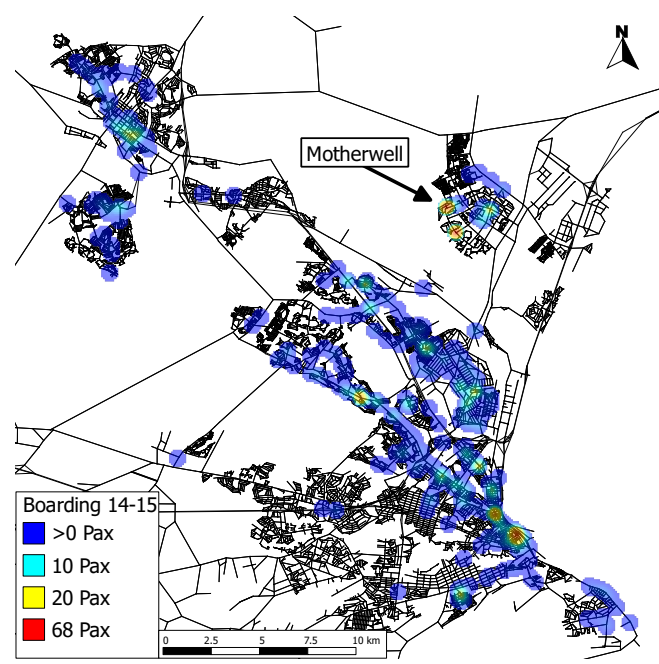

(c) Minibus boardings between 14 and 15

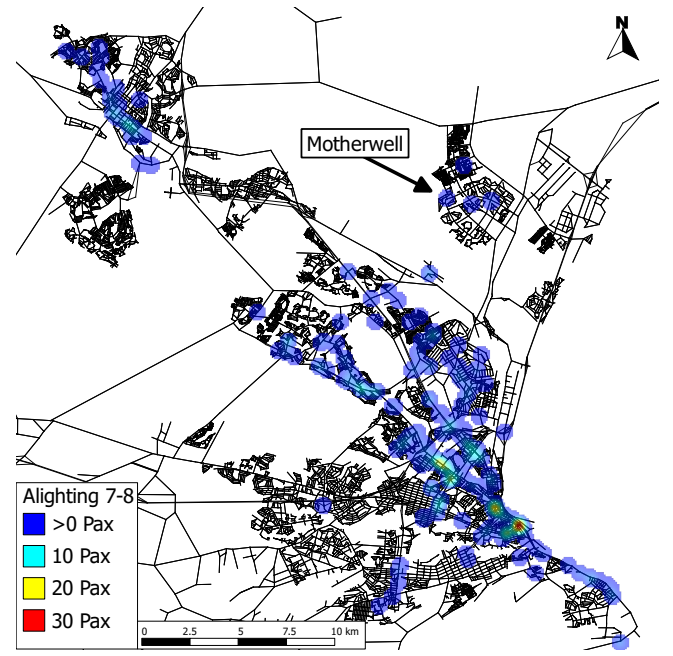

(b) Minibus alightings between 7 and 8

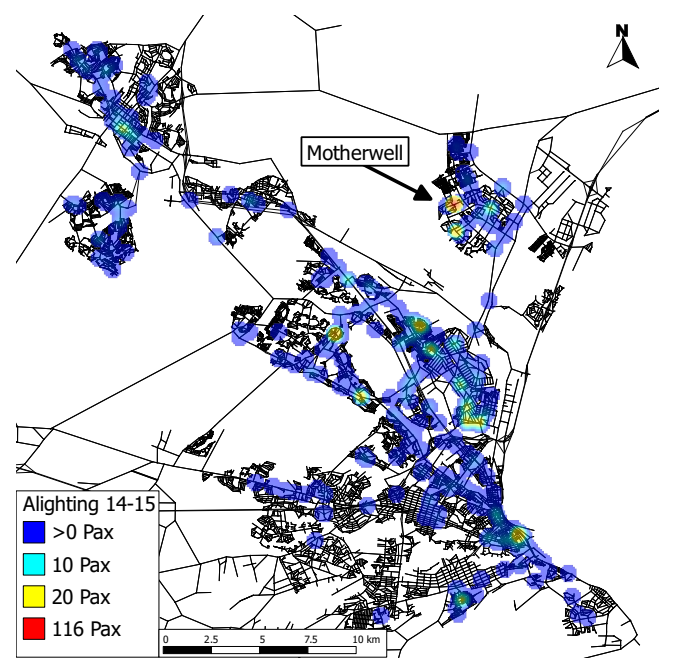

(d) Minibus alightings between 14 and 15

Figure 7: Boardings and alightings during the morning peak and the afternoon peak. Boardings and alightings induced by transfers are excluded.

separated clusters. In consequence, boarding activities of minibus users going to work in the morning concentrate at different locations than the corresponding alightings. For example, boarding activities during the morning peak (as shown in Figure 7a) tend to be scattered in residential areas, whereas alighting activities (Figure $7 \mathrm{~b}$ ) are more clustered and occur in areas with workplaces (see Figure 1 for comparison).

The analysis of the afternoon peak's boarding activities (Figure 7c) features the same hot spots as the alightings during the morning peak. In addition, there are a few new hot spots, especially in the Township of Motherwell in the northeast portion of the research area. These trips are related to the (primary) schools situated in that area. Since the scholars tend to live nearby and just walk to school, the minibus trips likely belong to school employees that are leaving the township when the school day ends. 


\subsection{Discussion}

The evolved minibus network analyzed in this section heavily depends on the configuration of the minibus model. The cost structure has an especially huge impact on the service quality of the minibuses. Given that the fare is set to a value experienced by the authors, the average load factor that a driver needs to cover his or her expenses derives directly from his or her running costs. As stated earlier in Section 2, it becomes the goal of the driver to earn the agreed upon check-in amount for the vehicle owner. Since little is known about these agreements, the authors had to rely on an educated guess regarding the costs that vehicle owners have to cover and thus what the drivers need to earn.

From transport planning studies conducted for the public transport authority of Berlin, Germany (see Neumann (2014)), the sensitivity of the minibus model toward changes of the cost structure is known. Higher fares or smaller running costs directly translate into smaller average load factors. This is a direct result of the vehicle owners always balancing their budgets to zero (see subsection 3.4). Thus, vehicle owners put more vehicles into service when serving the same amount of passengers. As a consequence, high-demand corridors are served more frequently, and the number of denied boardings due to vehicle capacity constraints decreases. Moreover, vehicle owners are able to provide more services in low-demand areas that could not previously cover the related costs. Effectively, this increases the total coverage of the minibus network and reduces access/egress walking distances.

\section{Conclusion and outlook}

The minibus model presented in this paper, we would claim, is able to create "close-to-reality" minibus networks in a South African context. The networks evolve according to the constraints of the input data given. In case detailed minibus data on routes and headways is available, the model can also be used to simulate a fixed minibus supply. In both cases, the minibus-specific behavior is integrated into an existing multi-modal transport model. Transport planners and policymakers can thus analyze the implications of policy measures such as the currently debated implementation of a minibus (taxi) subsidy.

Being able to realistically mimic the behavior, for the first time, of taxi stakeholders opens a number of opportunities for decision makers. This is possible because if the model encapsulates behavior, testing different scenarios may yield changes in behavior-be it by the driver (route variation), the owner (vehicle size and mix), or the association (route fares). What are some of the scenarios? When introducing a new formal bus rapid transit (BRT) line, resistance from the taxi industry has seen a few instances where the BRT lines are jointly operated, and owned, by the taxi industry (Joubert 2013). An ex-ante analysis of what impact the BRT line will have on the taxi industry may support negotiations on the number of taxi vehicles that will be removed from the line with the introduction of new high-capacity BRT vehicles.

Another example may be the introduction of road pricing, as is planned elsewhere in the country. The fee and discount structure of the toll-for example, a discount for public transport vehicles or time-of-day discounts-can be evaluated. Testing the fee structures in an agent-based setting can help determine how minibus taxi routes may evolve, how the fare structures change, and ultimately what the financial implications would be for commuters that already represent the low-income quartile. It may also assist in identifying unintended consequences that may, for example, be caused by newly congested areas created as a result of other road users diverting.

As far as reliable validation data is available, the network represents the coverage and main characteristics of the real minibus system of the Nelson Mandela Bay Municipality and reflects the minibus users' travel pattern. However, the model can be further enhanced to incorporate more travel behavior specific to South Africa. This includes ceasing service outside the peak hours and integrating vehicle holding strategies at the minibus routes' termini (taxi ranks) to reflect drivers' tendency to depart only 
with nearly fully loaded vehicles. Furthermore, future implementations should depict the fact that usually only one operator serves a certain relation. Another issue derives from the diversification of service types. While local and long-distance routes overlap, both services charge different fares; thus, minibus users are more willing to board a local minibus if it serves the same trip with equal or better quality. With the recent implementation of fares into MATSim's router architecture, this becomes possible and trips can be calculated with respect to the preferences of individual travelers. After implementing fares into the current model, a second step will be the implementation of a detailed decision modelbased on intrinsic motivation and personal income-to represent the agents reaction to changes of the system (e.g., to evaluate the influence and acceptance of new public transport systems).

\section{Acknowledgments}

This research was partially funded by the South African National Treasury. 


\section{References}

Arthur, B. 1994. Inductive reasoning, bounded rationality, and the bar problem. American Economic Review (Papers and Proceedings), 84:406-411.

Axelrod, R. 1984. The Evolution of Cooperation. NY: Basic Books.

Balmer, M., B. Raney, and K. Nagel. 2005. Adjustment of activity timing and duration in an agentbased traffic flow simulation. In H. Timmermans, ed., Progress in activity-based analysis, pp. 91-114. Oxford, UK: Elsevier.

Cervero, R. 2000. Informal Transport in the Developing World. HS/593/00E. UN-HABITAT.

Cervero, R. and A. Golub. 2007. Informal transport: A global perspective. Transport Policy, $14(6): 445-457$.

City of Johannesburg. 2004. Integrated Transport Plan 2003/2008. City of Johannesburg.

Cortés, C. E. 2003. High coveragepoint to point transit (HCPPT): A new design concept and simulationevaluation of operational schemes. Ph.D. thesis, University of California, Irvine.

DoT. 2005. The first South African national household travel survey 2003. Technical report, Department of Transport, Pretoria, South Africa.

Drossel, B. 2001. Biological evolution and statistical physics. Preprint arXiv:cond-mat/0101409v1, arXiv.org.

Dugard, J. 1996. Drive on? An analysis of the deregulation of the South African taxi industry and the emergence of the subsequent "taxi-wars". Master's thesis, University of Cambridge.

Dugard, J. 2001. From low intensity war to mafia war: Taxi violence in South Africa (1987-2000). In Violence and Transition Series, volume 4. The Centre for the Study of Violence and Reconciliation. Available online from http://www.csvr.org.za/docs/taxiviolence/fromlowintensity.pdf. Retrieved 15 June 2012.

ENEA. 2004. Demand responsive transport services: Towards the flexible mobility agency. Italian National Agency for New Technologies, Energy and the Environment.

Fernandez, J., J. de Cea, and R. Henry Malbran. 2008. Demand responsive urban public transport system design: Methodology and application. Transportation Research Part A-Policy And Practice, 42(7):951-972. ISSN 0965-8564. doi: 10.1016/j.tra.2007.12.008.

Hofbauer, J. and K. Sigmund. 1998. Evolutionary games and replicator dynamics. Cambridge University Press.

Jokinen, J., T. Sihvola, E. Hyytia, and R. Sulonen. 2011. Why urban mass demand responsive transport? In Integrated and Sustainable Transportation System (FISTS), 2011 IEEE Forum on, pp. 317-322. IEEE.

Joubert, J. W. 2013. Gauteng paratransit: Perpetual pain or potent potential. In T. Kuhnimhof, ed., Megacity Mobility Culture, Lecture Notes in Mobility, chapter 6, pp. 107-126. Springer-Verlag.

Khosa, M. 1994. Accumulation and labour relations in the taxi industry. Transformation, 24:5-71.

MATSim. 2013. Multi-Agent Transportation Simulation. http://www.matsim.org. URL http:// www.matsim.org.

McCaul, C. 1990. No easy ride: The rise and future of the black taxi industry. Technical report, South African Institute of Race Relations.

Müller, K. and K. W. Axhausen. 2011. Hierarchical IPF: Generating a synthetic population for Switzerland. In 51 ${ }^{\text {st }}$ ERSA Conference. doi: 10.3929/ethz-a-006620748.

Neumann, A. 2014. A paratransit-inspired evolutionary process for public transit network design. Ph.D. thesis, Technische Universität Berlin. urn:nbn:de:kobv:83-opus4-53866.

Neumann, A. and K. Nagel. 2010. Avoiding bus bunching phenomena from spreading: A dynamic approach using a multi-agent simulation framework. VSP Working Paper 10-08, TU Berlin, Transport Systems Planning and Transport Telematics. See www.vsp.tu-berlin.de/publications. 
Neumann, A. and K. Nagel. 2012. A paratransit-inspired evolutionary process for public transit network design. Annual Meeting Preprint 12-0716, Transportation Research Board, Washington D.C. Also VSP Working Paper 11-15, see www.vsp.tu-berlin.de/publications.

Neumann, A. and K. Nagel. 2013. Passenger agent and paratransit operator reaction to changes of service frequency of a fixed train line. Procedia Computer Science, 19(0):803-808. ISSN 18770509. doi: 10.1016/j.procs.2013.06.106.

OpenStreetMap. 2012. Map data () OpenStreetMap contributors, CC BY-SA. Available online from www.openstreetmap.org, URL http://www.openstreetmap.org.

Pagés, L., R. Jayakrishnan, and C. E. Cortés. 2006. Real-time mass passenger transport network optimization problems. In Network Modeling 2006, number 1964 in Transportation Research Record, pp. 229-237. 2101 Constitution Ave, Washington, DC 20418 USA: National Academy of Sciences. ISBN 978-0-309-09973-8.

Palmer, R., W. B. Arthur, J. H. Holland, B. LeBaron, and P. Tayler. 1994. Artificial economic life: a simple model of a stockmarket. Physica D, 75:264-274.

Pirie, G. H. 1992. Traveling under apartheid. In The Apartheid city and beyound: Urbanization and social change in South Africa. Routledge.

Raney, B. and K. Nagel. 2006. An improved framework for large-scale multi-agent simulations of travel behaviour. In P. Rietveld, B. Jourquin, and K. Westin, eds., Towards better performing European Transportation Systems, pp. 305-347. London: Routledge.

Rieser, M. 2010. Adding transit to an agent-based transportation simulation concepts and implementation. Ph.D. thesis, TU Berlin. Also VSP Working Paper 10-05, see www.vsp.tuberlin.de/publications.

Rieser, M. and K. Nagel. 2009. Combined agent-based simulation of private car traffic and transit. In Proceedings of The 12th Conference of the International Association for Travel Behaviour Research (IATBR). Jaipur, India. Also VSP Working Paper 09-11, see www.vsp.tu-berlin.de/publications, URL www.iatbr.org.

Roos, D. and D. Alschuler. 1975. Paratransit - existing issues and future directions. Transportation, $4(4): 335-350$.

Schalekamp, H. and R. Behrens. 2010. Engaging paratransit on public transport reform initiatives in South Africa: A critique of policy and an investigation of appropriate engagement approaches. Research in Transportation Economics, 29(1):371-378.

SSI Engineers and Environmental Consultants. 2011. Comprehensive Integrated Transport Plan 2011/12. Infrastructure and Engineering - Directorate Nelson Mandela Bay Municipality.

Venter, C. J. 2011. The lurch towards formalisation: lessons from the implementation of BRT in Johannesburg, South Africa. In Thredbo 12, International Conference Series on Competition and Ownership in Land Passenger Transport.

Woolf, S. E. and J. W. Joubert. 2013. A people-centred view of paratransit in South Africa. Cities, 35:284-293. doi: 10.1016/j.cities.2013.04.005. 